OPEN ACCESS

Edited by:

Tamer Demiralp,

Istanbul University, Turkey

Reviewed by:

Chiang-shan R. Li,

Yale University, United States

Dieter J. Meyerhoff,

University of California,

San Francisco, United States

*Correspondence:

Ferdinand Binkofski

fbinkofski@ukaachen.de

Received: 10 November 2018

Accepted: 22 May 2019

Published: 13 June 2019

Citation:

Alkhasli I, Sakreida K, Mottaghy FM and Binkofski F (2019) Modulation of

Fronto-Striatal Functional

Connectivity Using Transcranial

Magnetic Stimulation.

Front. Hum. Neurosci. 13:190.

doi: 10.3389/fnhum.2019.00190

\section{Modulation of Fronto-Striatal Functional Connectivity Using Transcranial Magnetic Stimulation}

\author{
Isabel Alkhasli ${ }^{1}$, Katrin Sakreida ${ }^{2}$, Felix M. Mottaghy ${ }^{3,4,5}$ and Ferdinand Binkofski ${ }^{1,5,6 *}$ \\ ${ }^{1}$ Section Clinical Cognitive Sciences, Department of Neurology, Faculty of Medicine, RWTH Aachen University, Aachen, \\ Germany, ${ }^{2}$ Department of Neurosurgery, Faculty of Medicine, RWTH Aachen University, Aachen, Germany, ${ }^{3}$ Department \\ of Nuclear Medicine, Faculty of Medicine, RWTH Aachen University, Aachen, Germany, ${ }^{4}$ Department of Radiology and \\ Nuclear Medicine, Maastricht University Medical Center (MUMC+), Maastricht, Netherlands, ${ }^{5} \mathrm{Juelich}$ Aachen Research \\ Alliance (JARA) - BRAIN, Juelich, Germany, ${ }^{6}$ Research Centre Juelich, Institute of Neuroscience and Medicine (INM-4), \\ Juelich, Germany
}

Background: The fronto-striatal network is involved in various motor, cognitive, and emotional processes, such as spatial attention, working memory, decision-making, and emotion regulation. Intermittent theta burst transcranial magnetic stimulation (iTBS) has been shown to modulate functional connectivity of brain networks. Long stimulation intervals, as well as high stimulation intensities are typically applied in transcranial magnetic stimulation (TMS) therapy for mood disorders. The role of stimulation intensity on network function and homeostasis has not been explored systematically yet.

Objective: In this pilot study, we aimed to modulate fronto-striatal connectivity by applying iTBS at different intensities to the left dorso-lateral prefrontal cortex (DLPFC). We measured individual and group changes by comparing resting state functional magnetic resonance imaging (rsfMRI) both pre-iTBS and post-iTBS. Differential effects of individual sub- vs. supra-resting motor-threshold stimulation intensities were assessed.

Methods: Sixteen healthy subjects underwent excitatory iTBS at two intensities [90\% and $120 \%$ of individual resting motor threshold (rMT)] on separate days. Six-hundred pulses (2 s trains, $8 \mathrm{~s}$ pauses, duration of $3 \mathrm{~min}, 20 \mathrm{~s}$ ) were applied over the left DLPFC. Directly before and 7 min after stimulation, task-free rsfMRI sessions, lasting 10 min each, were conducted. Individual seed-to-seed functional connectivity changes were calculated for 10 fronto-striatal and amygdala regions of interest with the SPM toolbox DPABI.

Results: Sub-threshold-iTBS increased functional connectivity directly between the left DLPFC and the left and right caudate, respectively. Supra-threshold stimulation did not change fronto-striatal functional connectivity but increased functional connectivity between the right amygdala and the right caudate.

\footnotetext{
Abbreviations: rsfMRI, Resting state functional magnetic resonance imaging; TMS, Transcranial magnetic stimulation; iTBS, Intermittent theta burst stimulation; ANOVA, Analysis of variance; rMT, Resting motor threshold; DLPFC, Dorso-lateral prefrontal cortex; BOLD, Blood oxygenation level dependent.
} 
Conclusion: A short iTBS protocol applied at sub-threshold intensities was not only sufficient, but favorable, in order to increase bilateral fronto-striatal functional connectivity, while minimizing side effects. The absence of an increase in functional connectivity after supra-threshold stimulation was possibly caused by network homeostatic effects.

Keywords: functional connectivity, prefrontal cortex, DLPFC, striatum, intermittent theta burst stimulation (iTBS), resting state, fronto-striatal network

\section{INTRODUCTION}

The fronto-striatal network is known to play a significant role in various motor, cognitive, and emotional processes (Breitenstein et al., 1998; Arnsten, 2009; Beste et al., 2012). The striatum, which is comprised of caudate, putamen, and the nucleus accumbens, receives afferents from the substantia nigra and the ventral tegmentum in the midbrain, and in turn projects to the pallidum, thalamus, globus pallidus, and substantia nigra. Additionally, there are strong output connections to the amygdala, hypothalamus, and pedunculopontine nucleus (Robbins and Everitt, 1992). Animal studies have suggested a direct modulation mechanism of the prefrontal cortex (PFC) through its projections onto the striatal neurons (Bouyer et al., 1984; Sesack and Pickel, 1992; Murase et al., 1993; Karreman and Moghaddam, 1996; Keck et al., 2002; Kanno et al., 2003, 2004). More recently, neuroimaging studies done on human subjects identified strong anatomical and functional connections between the dorsolateral PFC (DLPFC) and the dorsal-posterior caudate, while the ventrolateral PFC was found to be mainly interconnected with the ventral caudate (Leh et al., 2007; Di Martino et al., 2008; Choi et al., 2012). Functionally, the DLPFC is associated with a wide range of executive functions such as working memory, selective attention and decision making (Curtis and D'Esposito, 2003; Glenn et al., 2009) while the ventrolateral PFC is more involved in motor control (Levy and Wagner, 2011).

Resting state functional magnetic resonance imaging (rsfMRI) research is a powerful tool to reveal intrinsically, functionally connected areas by simply correlating the ongoing resting blood oxygenation level dependent (BOLD) activity of anatomically distinct areas. Areas that with synchronized ongoing activation are thought to be functionally linked. RsfMRI studies of the fronto-striatal network, as well a task-based fMRI research have, for example, been used to reveal the dysfunction of this system, which can lead to severe cognitive and behavioral, as well as emotional symptoms. Abnormal fronto-striatal network functions have been linked to neurodegenerative diseases such as Parkinson's disease and prodromal Alzheimer dementia, as well as mood disorders and impulse-control disorders. They can cause symptoms ranging from aggression, mania, and impulsiveness, to anhedonia, depression, and attention-deficits (Wessa et al., 2007; Menzies et al., 2008; Heller et al., 2009; Cubillo et al., 2010; Courtney et al., 2013; Wang et al., 2013; Salomons et al., 2014; Baggio et al., 2015).
In healthy subjects, acute and chronic stress can impair the structure and function of the entire fronto-striatal and frontolimbic system (Arnsten, 2009). In this context, the amygdala is particularly important, because it is highly interconnected with these systems and is a key element in emotional processing and regulation. Functional connectivity between the amygdala and the PFC was found to be increased during emotional self-regulation tasks (Banks et al., 2007). Moreover, increased dopamine levels in the amygdala are associated with aggressiveness (Phelps and LeDoux, 2005).

Combining positron-emissions-tomography (PET) and transcranial magnetic stimulation (TMS), Strafella et al. (2001, 2003) were the first to demonstrate, that the functional modulation of the lateral frontal (Strafella et al., 2001) and the primary motor cortex (Strafella et al., 2003) via TMS has a significant effect on the dopamine release in the ipsilateral caudate (Strafella et al., 2001) and putamen (Strafella et al., 2003), as measured by a radiolabeled D2-receptor ligand. They used a repetitive TMS protocol of $10 \mathrm{~Hz}$ lasting $30 \mathrm{~min}$ and a stimulation intensity of $100 \%$ of the individual resting motor threshold (rMT).

TMS to the PFC is now increasingly used as a treatment tool for major depression and bipolar affective disorder (Johnson et al., 2013; Janicak and Dokucu, 2015). The rationale for using excitatory TMS to the left PFC is an imbalance in activity between the right and left PFC in these disorders. The lower activity of the left PFC can be enhanced using TMS. In these studies, stimulation duration is often very long, and stimulation intensity is set at or rather above of the individual rMT, which can induce high levels of pain or discomfort.

Intermittent theta burst transcranial magnetic stimulation (iTBS) has been shown to reliably increase regional excitability as well as functional connectivity between brain areas (Huang et al., 2005). Studies comparing more conventional TMS protocols with more recently introduced TBS protocols collectively found a comparable effectiveness in changing neuronal excitability (Zafar et al., 2008; Ziemann et al., 2008). Combining long-lasting effects on local and network activity with minimal stimulation time iTBS is a promising therapeutic tool for disorders of the fronto-striatal system (Brunelin et al., 2011; Li C.-T. et al., 2014; Bakker et al., 2015; Duprat et al., 2016). These studies typically apply iTBS to the PFC and measure a behavioral outcome variable through a standardized test. In a similar study on Parkinson patients (Benninger et al., 2011) found beneficial effects of iTBS on mood, but no improvement in other disease-related measures. 


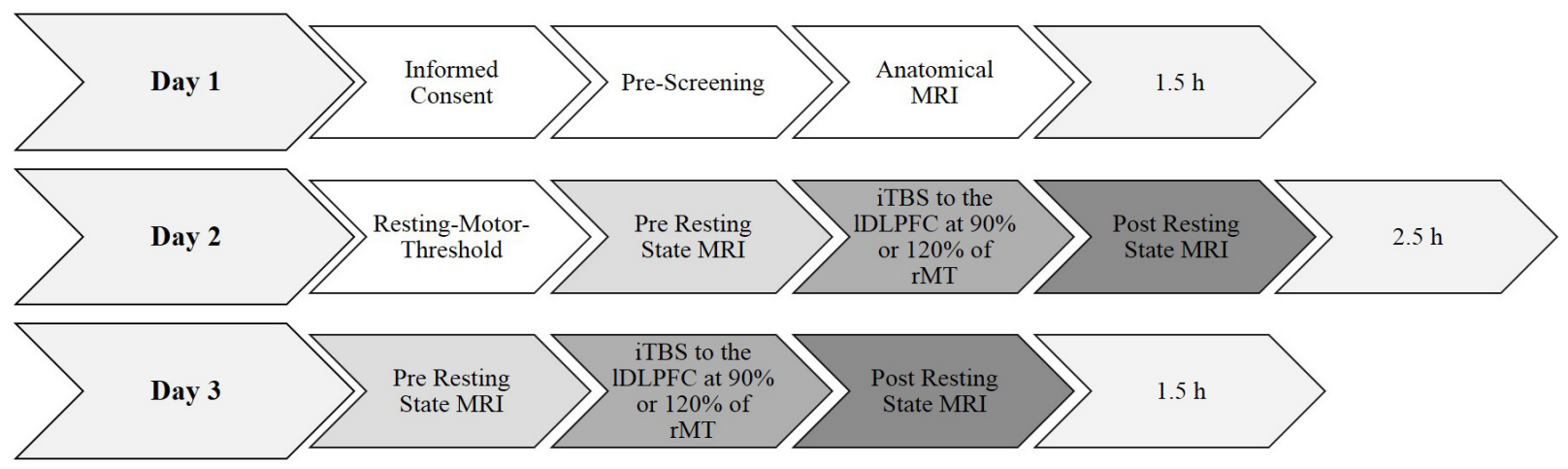

FIGURE 1 | Summary of the experimental design and durations. Day 1 and day 2 took $1.5 \mathrm{~h}$ each. Determining the resting-motor threshold took approximately $45 \mathrm{~min}$. All anatomical and functional magnetic resonance imaging (fMRI) scans took approximately 10 min each. The experimental procedure took in total approximately $5.5 \mathrm{~h}$ per participant. rMT, resting motor threshold.

Nevertheless, the precise mechanisms of TMS-induced network modulations and the role of stimulation intensity has not been explored systematically and in detail yet. Therefore, we applied iTBS to the left PFC using sub and supra-restingmotor-threshold stimulation intensities to test parameters which combine strong network modulation and minimal side effects. The aim of this pilot study was to compare the effect of sub- vs. supra-threshold iTBS on functional connectivity of the entire fronto-striatal network by conducting resting state fMRI measurements before and after the iTBS. Additionally, we wanted to explore functional connectivity between the frontostriatal network and the amygdala because of its importance for emotional-processing. Portions of the data published in this article have previously been presented as conference posters (Effnert et al., 2016a,b).

\section{MATERIALS AND METHODS}

\section{Participants}

Our study was approved by the local ethical committee (protocol number: 003/15), and procedures involving human participants were in accordance with the ethical standards of the institutional and/or national research committee and with the 1964 Helsinki Declaration and its later amendments or comparable ethical standards. Informed consent was obtained from all individual participants included in the study.

Sixteen neurologically and mentally healthy, right handed (validated by the Edinburgh Handedness Inventory, Oldfield, 1971) participants were recruited (mean age $=27.63, \mathrm{SD}=6.95$; 8 males). Participants were pre-screened for TMS and MRI exclusion criteria. Sensitivity to the TMS protocol was investigated prior to the experiment by applying the excitatory iTBS protocol to the dedicated prefrontal location and increasing the stimulation intensity stepwise to a maximum of $50 \%$ of the maximum stimulator output. Subjects could then decide whether or not the stimulation was too unpleasant and whether they still wanted to participate.

\section{Experimental Procedure}

A summary of the experimental procedure and the durations are shown in Figure 1. Each participant was invited into the laboratory on three separate days. The pre-selection procedure and informed consent were done on day 1 . On day 2, the individual rMT was determined using a standardized protocol (Rossi et al., 2009; Rossini et al., 2015; see "Transcranial Magnetic Stimulation" section below). After a break of about $20 \mathrm{~min}$, a $10 \mathrm{~min}$ baseline resting state MRI measurement was collected. For the following TMS measurement, the participant was brought outside the scanner room lying supine on the mobile scanner bed. The participants were registered with their anatomical data (see "Magnetic Resonance Imaging" section below for scanning parameters) and the iTBS lasting $3 \mathrm{~min}$ and $20 \mathrm{~s}$ was applied over the left PFC either at $90 \%$ or at $120 \%$ of their rMT. The order of the $90 \%$ and the $120 \%$ stimulation intensities was counterbalanced and alternated between subjects. After a 7-min-break the participants were rolled into the scanner again, lying in an unchanged position on the scanner bed and the 10-min-post resting state MRI measurement was conducted. During the scan participants were shown a small black fixation spot in the middle of a gray background. They were instructed to fixate the dot at all times, to relax, do not fall asleep, lie as still as possible, and to try not to think of anything in particular. The measurements on day 3 were conducted identically to day 2 by only varying stimulation intensity. The rMT was not determined again.

\section{Transcranial Magnetic Stimulation}

To determine the individual rMT, an anatomical T1 MRI scan was used to locate the hand area of the left motor cortex. The subjects were placed in a comfortable chair and then their heads were registered to their individual anatomical T1 scan using neuronavigation software (TMS navigator, Localite GmbH, Sankt Augustin, Germany). The presumed hand area was identified visually through anatomical landmarks and stimulated with biphasic single pulses using a figure-of-eight coil (MagVenture C-B60) connected to a MagPro stimulator 
(X100 MagVenture, Farum, Denmark). Electrodes were fitted to the participant's right index-finger and motor evoked potentials were monitored. Stimulation intensity was first increased in $2 \%$ steps until the hand area could be determined through a clear supra-threshold $(>50 \mu \mathrm{V})$ motor evoked potential. Intensity was then reduced stepwise to find the lowest intensity to still induce a supra-threshold motor evoked potential.

The experimental excitatory iTBS (Huang et al., 2005) protocol consisted of 600 pulses spaced-out over $3 \mathrm{~min}$ and 20 s. It was comprised of 20 trains and 10 theta-bursts and was applied over the left DLPFC. Between each of the $2 \mathrm{~s}$-long trains $(50 \mathrm{~Hz})$ there was an $8 \mathrm{~s}$ long pause. The stimulation site was determined by transforming the individual anatomical images into the Talairach system using the neuronavigation system (Localite TMS navigator) and marking the Talairach coordinates $\mathrm{x} / \mathrm{y} / \mathrm{z}=-45 / 45 / 35$ as stimulation target. Additionally, individual anatomical landmarks (inferior and superior frontal sulcus) were taken into consideration to correct the location of the stimulation side in case the coordinate was outside of the DLPFC. This procedure was applied as suggested by Fitzgerald et al. (2009). They were able to demonstrate that the use of this neuro-navigational method to target a PFC site enhanced response to TMS treatment in depression, as compared to the formerly standard $5-\mathrm{cm}$ procedure (locating the hand areal and simply measuring $5 \mathrm{~cm}$ in the sagittal plane on the scalp). The mean Talairach coordinates of the actual stimulation sites were $\mathrm{x} / \mathrm{y} / \mathrm{z}=-41 / 37 / 31(S D=5.33 / 14.8 / 9.83)$. The mean $\mathrm{rMT}$ was $43 \%(S D=4.82)$ of the maximum stimulator output. The mean sub-threshold stimulation applied was $38 \%(S D=3.91)$ and the mean supra-threshold was $50 \%(S D=5.39)$ of the maximum stimulator output.

\section{Magnetic Resonance Imaging}

MRI scans were measured on a Magnetom Prisma $3.0 \mathrm{~T}$ whole-body scanner (Siemens Medical Solutions, Erlangen, Germany). Anatomical data was acquired using a threedimensional magnetization-prepared, rapid acquisition gradient-echo sequence (MPRAGE) with the following parameter: 300 repetitions, $\mathrm{TR}=2,300 \mathrm{~ms}, \mathrm{TE}=2.98 \mathrm{~ms}, 9^{\circ} \mathrm{flip}$ angle, $\mathrm{FOV}=256 \mathrm{~mm}, 176$ sagittal slices, slice thickness $=1 \mathrm{~mm}$ and in-plane resolution $=1 \times 1 \times 1 \mathrm{~mm}$.

Resting state MRI data were measured with a gradient echo planar imaging (EPI) sequence with the following parameters: $\mathrm{TR}=2,000 \mathrm{~ms}, \mathrm{TE}=28 \mathrm{~ms}, 77^{\circ}$ flip angle, $\mathrm{FOV}=192 \mathrm{~mm}$, 34 axial slices (interleaved acquisition), $3 \mathrm{~mm}$ slice thickness, EPI volumes and in-plane resolution $=3 \times 3 \times 3 \mathrm{~mm}$. Each of the sequences lasted about $10 \mathrm{~min}$.

\section{Pre-processing of Resting State Data and Functional Connectivity}

MRI-data was analyzed using the Statistical Parametric Mapping software SPM10 (Welcome Department of Cognitive Neurosciences, London, UK) and Data Processing and Analysis for Resting-State Brain Imaging (DPABI, Yan et al., 2016) toolboxes running under Matlab R2012b (MathWorks Inc., Natick, MA, USA). Pre-processing of the rsfMRI data included the following steps: removal of first five volumes to discard saturation effects, slice time correction, realignment, segmentation, nuisance covariates regression with white matter and cerebrospinal fluid as regressors, head motion correction, head motion scrubbing as regressors, band pass filtering of the frequencies $0.01-0.08 \mathrm{~Hz}$, spatial smoothing ( $5 \mathrm{~mm} \mathrm{FWHM}$ ) and detrending (removal of gradual shifts). The root-mean-square of the head motion translation parameters [displacement $=$ square root $\left.\left(\mathrm{x}^{2}+\mathrm{y}^{2}+\mathrm{z}^{2}\right)\right]$ across all participants and sessions was $0.43 \mathrm{~mm}$, with a session mean maximum of $1.44 \mathrm{~mm}$ and minimum of $0.15 \mathrm{~mm}(S D=0.27 \mathrm{~mm})$.

Seed-to-seed functional connectivity was calculated using the following 10 seed regions: individual stimulation site (sphere of $1 \mathrm{~cm}$ diameter, left DLPFC), right DLPFC, left and right caudate, left and right nucleus accumbens, left and right putamen, as well as left and right amygdala. For each region a left-side and a right-side seed were calculated individually. Three-dimensional seed masks were obtained from each participant's individual T1-anatomy and then co-registered with the corresponding functional data. These masks were then used to extract a mean BOLD signal calculated from the time series of all mask voxels within the particular seed. Functional connectivity is thus the correlation between the mean BOLD signals of two areas. All correlation values are Fisher-Z-transformed. The resulting correlation maps were co-registered to the MNI standard template.

All further statistical processes were done using SPSS Statistics 23 (IBM Corp., Armonk, NY, USA). To address the question of whether the TMS stimulations modulated fronto-striatal connectivity, a mean BOLD signal was calculated for the entire striatum. This was done by averaging the mean signals of all above mentioned striatal seeds (caudate, nucleus accumbens and putamen). Fisher-Z-transformed correlations (i.e., functional connectivity) between this big striatal seed and the stimulation site seed (left DLPFC) were then calculated for each of the four measurements $(90 / 120 \% \times$ pre/post). This approach was chosen to avoid over-representation of the CA signals since this region comprises the biggest part of the striatum and thus the most voxels.

\section{RESULTS}

A $2 \times 2$ repeated measures analysis of variance (ANOVA) of the functional connectivity between the left DLPFC and the entire striatum revealed a significant interaction $\left(F_{(1,15)}=11.29\right.$, $\left.p=0.004, \eta_{\mathrm{p}}^{2}=0.43\right)$ between the two factors intensity $(90 \%$ vs. $120 \%$ ) and time point (PRE vs. POST). This effect size and observed power of 0.88 indicate a large effect (Cohen, 2013). a priori paired $t$-tests comparing the pre and post functional connectivity values for each intensity separately, revealed that the mean fronto-striatal functional connectivity was significantly increased after the $90 \%$-stimulation (pre: $M=0.22, S D=0.24$; post: $M=0.35, S D=0.22 ; p=0.006)$, but not after the $120 \%$-stimulation (pre: $M=0.25, S D=0.26$; post: $M=0.20$, $S D=0.26 ; p=0.27$ ). Values are visualized in Figure 2 and summarized in Table 1. In both intensity conditions, functional connectivity was always positive and slightly decreases to a positive value closer to 0 in the $120 \%$ condition. Thus, the results 


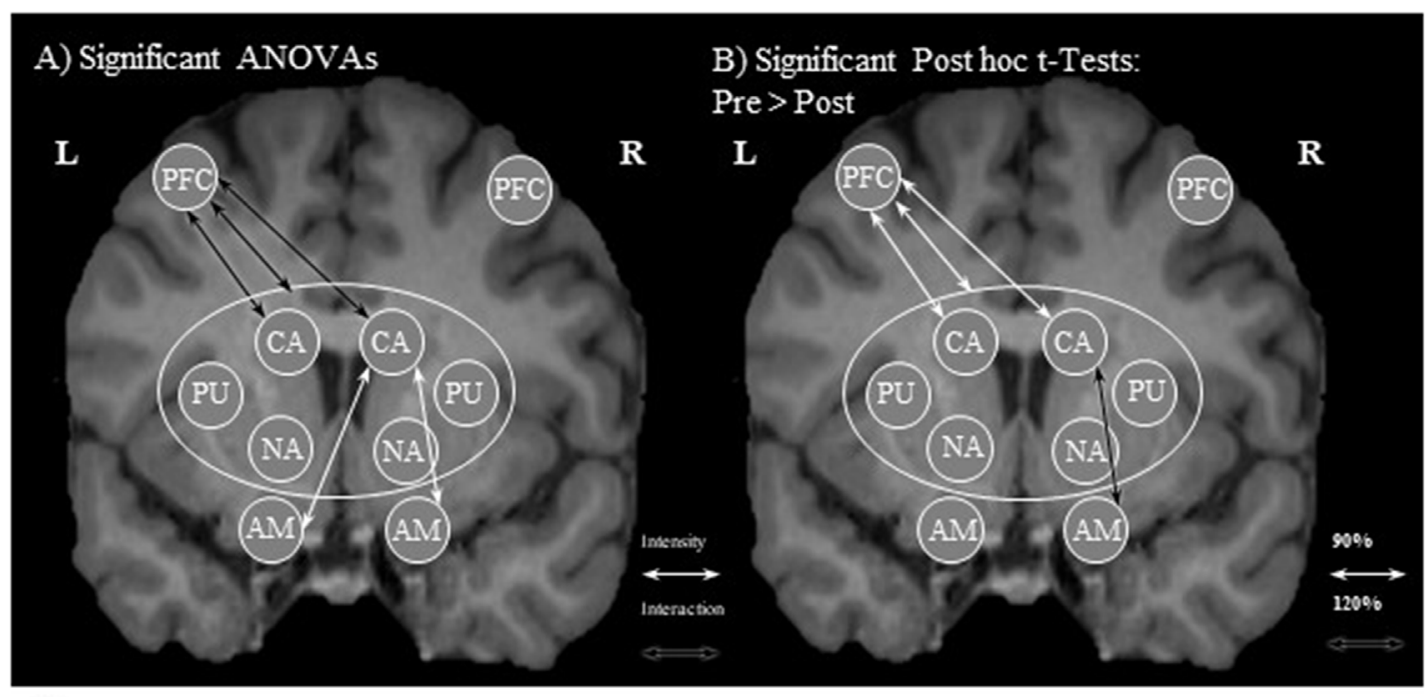

C)

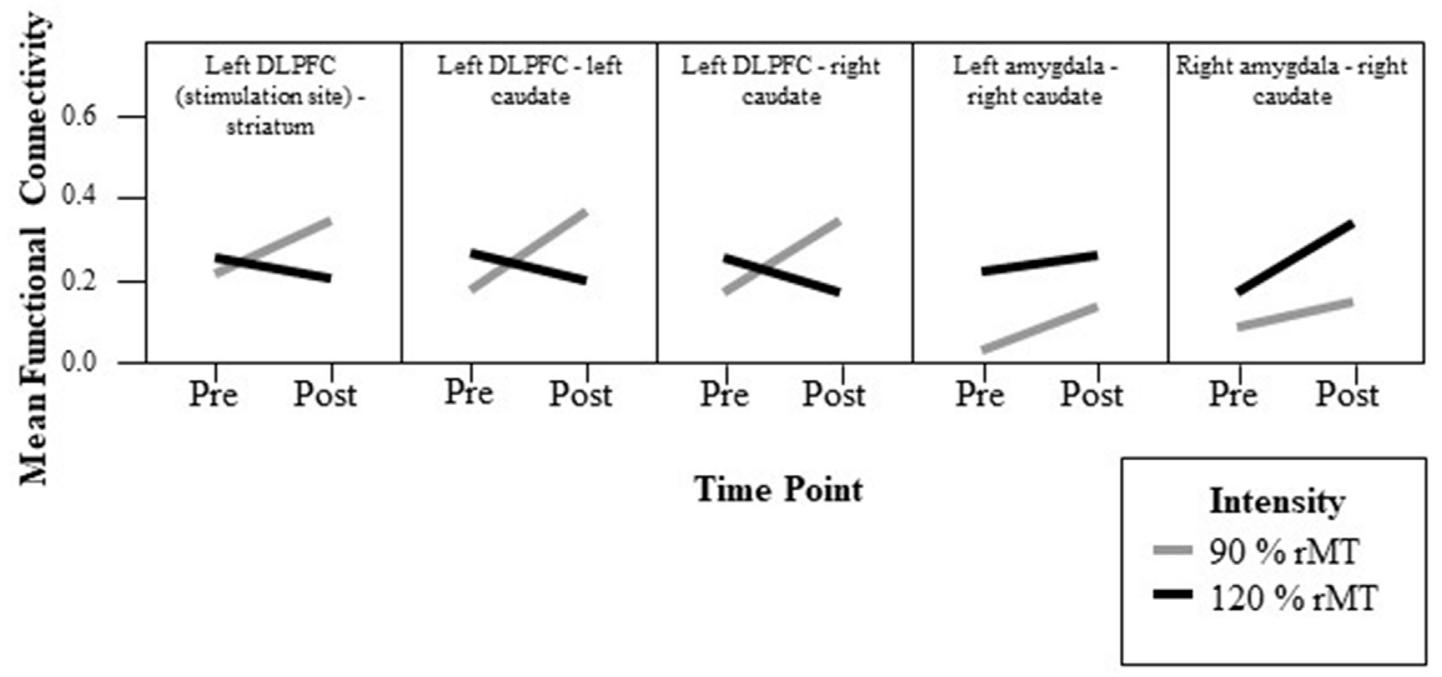

FIGURE 2 | Visualization of results. (A,B) Each arrow represents a significant test result. Significant analysis of variance (ANOVA) effects are shown in (A) and significant paired $t$-tests in (B). It can be seen that functional connectivity between the left prefrontal cortex (PFC; stimulation site) and the left and right caudate, respectively, as well as between the right caudate and the left and right amygdala, respectively, was modulated by the experimental stimulation. (A) White arrows indicate a significant intensity effect and black arrows a significant interaction effect. White ellipses indicate a seed region of interest. (B) Only pre-post transcranial magnetic stimulation (TMS) comparisons were calculated. All significant changes were increases from pre to post. Functional connectivity increased significantly only in the $90 \%$ condition between the stimulation site and the striatum. Analyzing the functional connectivity patterns between the stimulation site and each of the subregions of the striatum revealed that only the increase in functional connectivity between the stimulation site and the right and left caudate, respectively was significant. Additionally, functional connectivity between the right amygdala and the right caudate was increased only in the $120 \%$ condition. (C) Mean functional connectivity results. Shown are mean Fisher-Z-transformed correlations for the significant ANOVAs of functional connectivity between seeds, in the four different conditions $90 \%$ pre and post (gray line) and $120 \%$ pre and post (black line) measurements. Positive values indicate a positive coupling of signals between the two seeds. The first graph visualizes results between the stimulation site (left DLFC) and the entire striatum [consisting of left and right caudate (CA), left and right nucleus accumbens (NA), as well as left and right putamen (PU)]. AM, amygdala.

demonstrate a decoupling of frontal and striatal activity, rather than negative coupling.

To further explore the effect of the stimulation, a correlation matrix of the 10 seeds was created for each subject and each of the four conditions. These seed-to-seed-functional connectivity values were then subjected to repeated measures ANOVAs, resulting in 45 separate tests. The resulting multiple comparison problem was addressed by controlling the false discovery rate (FDR) using the Benjamini and Hochberg procedure (Benjamini and Hochberg, 1995). Planned post hoc paired $t$-tests were conducted to compare the pre vs. post iTBS measures only and $p$-values were adjusted using Bonferoni correction $(p=0.05 / 2)$. All significant results are summarized in Table 1, Figure 2. 
TABLE 1 | Statistically significant seed-to-seed functional connectivity (FC) results

\begin{tabular}{|c|c|c|c|c|c|c|c|c|c|}
\hline Seeds/Intensity & Effect/Post Hoc Pair & ss & $d f$ & MS/MD & $F / t$ & $p$ & Adj. $p$ & $\eta_{\mathrm{p}}^{2}$ & Obs. Power \\
\hline IDLPFC-STR & Interaction & 0.13 & 1 & 0.13 & 11.29 & 0.004 & - & 0.43 & 0.88 \\
\hline $90 \%$ & Pre-Post & - & 15 & -0.13 & -3.22 & 0.006 & - & - & - \\
\hline ICA-IDLPFC & Interaction & 0.23 & 1 & 0.23 & 29.93 & $<0.001$ & 0.003 & 0.67 & 0.999 \\
\hline $90 \%$ & Pre-Post & - & 15 & -0.18 & -4.15 & 0.001 & - & - & - \\
\hline rCA-IDLPFC & Interaction & 0.26 & 1 & 0.26 & 14.87 & 0.002 & 0.035 & 0.50 & 0.95 \\
\hline $90 \%$ & Pre-Post & - & 15 & -0.17 & -3.13 & 0.007 & - & - & - \\
\hline IAM-rCA & Intensity & 0.40 & 1 & 0.40 & 28.18 & $<0.001$ & 0.005 & 0.65 & 0.999 \\
\hline rAM-rCA & Intensity & 0.31 & 1 & 0.31 & 16.94 & 0.001 & 0.020 & 0.53 & 0.97 \\
\hline $120 \%$ & Pre-Post & - & 15 & -0.17 & -4.05 & 0.001 & - & - & - \\
\hline
\end{tabular}

Note. Results of significant ANOVAs and paired t-tests. Significant a priori t-test results are shown below each ANOVA row. Significance levels and observed power were computed using alpha $=0.05$ (two-tailed). Negative $t$-values indicate an increase in functional connectivity and positive t-values a decrease from pre to post. IAM/rAM, left/right amygdala; ICA/rCA, left/right caudate; IDLPFC/rDLPFC, left/right prefrontal cortex; STR, whole striatum; SS, sum of squares; df, degrees of freedom; MS, mean squares; MD, mean difference; F, F-ratio; $t$, t-value; p, p-value; Adj. p, FDR (Benjamini and Hochberg) adjusted p-value, $\eta_{p}^{2}$, partial eta square; Obs. Power, observed power.

This analysis revealed that the iTBS was able to modulate mainly fronto-caudal, as well as caudal-amygdala functional connectivity. Functional connectivity between the left-DLPFC and the bilateral caudal nuclei showed an interaction effect of the two factors time point of measurement and stimulation intensity, that matched the pattern of the whole-striatum analysis (see Figure 2C). This is functional connectivity increased from pre to post iTBS only in the $90 \%$ condition, but not in the $120 \%$ condition. Caudal-amygdala connectivity, on the other hand, showed a significant intensity effect only in the $120 \%$ condition, i.e., functional connectivity was higher in the $120 \%$ condition regardless of the time point of the measurement. Post hoc tests revealed a significant increase in functional connectivity between the pre and the post iTBS measurement for the right caudate and the right amygdala.

\section{DISCUSSION}

The aim of this study was to explore the effects of excitatory prefrontal iTBS at intensities below and above the rMT on the functional connectivity of the fronto-striatal brain network. Functional connectivity is a concept to quantify the synchronization i.e., correlation between brain areas as measured by correlation of BOLD signals. The frontal-cortical and striatal areas build a highly connected network that communicates through synchronization of neuronal activity. Low synchronization of activity and thus disrupted communication between those brain areas and desynchronization of activity can lead to aforementioned behavioral and emotional problems, but can also be induced externally to purposefully alter brain activity. This can, for example, be done by TMS.

The results of this study demonstrate the importance of choosing an optimal stimulation intensity when applying TMS in research or therapy. Differences in intensity led to significantly different functional connectivity patterns. The sub-threshold stimulation ( $90 \% \mathrm{rMT}$ ) was sufficiently high to directly strengthen connectivity of the left DLPFC and the caudate, which was not found in the $120 \%$ intensity condition.

Even though the exact mechanisms are still largely unclear (Ziemann and Siebner, 2015), it is empirically well established that certain TMS protocols can cause long-term-potentiation or depression-like plasticity (Ziemann et al., 2008). However, an increase in resting-state connectivity does not automatically indicate an increase in activation of one area, but rather an increase in BOLD pattern synchronization of connected areas. Nevertheless, several studies have found a direct linear relationship between the two measures (see for example Bohning et al., 2000; Nahas et al., 2001; Bestmann et al., 2005). Nahas et al., 2001, for example, delivered short $1 \mathrm{~Hz}$ trains of prefrontal TMS at $80 \%, 100 \%$ and $120 \%$ of the individual rMT, and found increased activation of the auditory cortex in all three conditions, contralateral prefrontal activity in the $100 \%$ condition and bilateral prefrontal activity in the $120 \%$ condition. Higher stimulation intensity was associated with higher BOLD signal under the coil and contra-laterally.

It is thus reasonable to interpret the changes in functional connectivity in the $90 \%$ condition in our study as a sign for increased network synchronization, caused by increased activity of the PFC. Nevertheless, the precise relationship between BOLD-signal changes and changes in functional connectivity is not straightforward and seems to rely highly on the involved areas and networks (Fox et al., 2012).

Interestingly, in the $120 \%$ condition, a very different pattern emerged. Bilateral amygdala activity was synchronized with the activity of the right caudate. The amygdala is not directly connected with the caudate (Robinson et al., 2012), thus caudate amygdala synchronization may be driven by shared regions, including those in the midbrain (Zhang et al., 2016) and the basal forebrain (Li C.-S. R. et al., 2014).

The lack of an increase of fronto-striatal functional connectivity in the $120 \%$ condition could possibly be explained by homeostatic mechanisms, that decrease activation and/or connectivity above a certain threshold (i.e., through surround inhibition). Stronger stimulation could induce stronger surround inhibition at the stimulation side in the PFC and connected areas. The mechanism of surround inhibition is well described in the sensory or the motor system, where it sharpens sensation or facilitates the selection of voluntary movements (Aungst et al., 2003; Sohn and Hallett, 2004; Angelucci et al., 2017). 
Additionally, it is known that the state of the brain during TMS is crucial for the modulation effect. A similar, seemingly paradoxical TMS effect has, for example, been reported for the preconditioning with different transcranial direct current stimulation (tDCS) protocols. Lang et al. (2004) and Siebner et al. (2004), for example, applied excitatory and inhibitory tDCS to the motor cortex and found that the modulatory effect of a following TMS on the cortical excitability was unexpectedly increased through an inhibitory preconditioning and decreased for the excitatory tDCS. Interestingly, this pattern was not changed by the variation of the TMS protocol. Both inhibitory and excitatory TMS produced the same modulation pattern. One should, however, keep in mind that the cytoarchitecture of the PFC is vastly different to that of the primary motor and sensory cortices and it is unknown whether these phenomena can also be observed in the PFC. Thus, it is important to conduct more detailed research on the effects of magnetic stimulation on the PFC.

Even though the DLPFC was chosen as the target stimulation area in our study, because of its special role in cognition, other cortical areas such as the pre-supplementary motor areas show strong connections to the striatal structures as well (Zhang et al., 2011). It might thus be an equally good candidate as a stimulation site to enhance striatal activity. TMS is already used as a therapeutic tool. Its usefulness has mainly been proven for mood disorders, such as depression or bipolar disorder (see for example Johnson et al., 2013; Janicak and Dokucu, 2015). However, stimulation intensity and other protocol parameters are usually chosen rather randomly. These studies have typically used long continuous stimulation protocols of up to $20 \mathrm{~min}$. Our study underlines the effectiveness of the comparatively short iTBS protocol of only $3.33 \mathrm{~min}$, as previously shown by Huang et al. (2005). ITBS could potentially become even more beneficial for other disorders involving the dysfunction of the frontostriatal system, such as Parkinson's disease or prodromal Alzheimer dementia. The ultimate goal for research and clinical application should thus be to find reliable ways to individually determine the lowest possible and highest necessary stimulation intensity and the optimal protocol to induce desired changes in brain activity.

\section{Limitations}

We did not systematically record perceived pain levels since participants were pre-screened to show a high tolerance against pain or discomfort during the TMS. This selection process could admittedly be prone to bias the sample. Inclusion of all participants and a systematic recording of subjective pain levels, on the other hand, would cause ethical and practical problems, and increase the issue of pain as a confounder. It has been reported that stress can impair the signaling pathway of the PFC (Arnsten, 2009). The influence of stress could indeed also explain the desynchronization of the fronto-striatal network in the $120 \%$ condition, as well as the increased amygdala activity. We did not implement a sham stimulation control group, because previous research in our lab has shown that participants can tell the difference between a sham and a real stimulation very well. We felt that the pre-measurement taken immediately before each of the stimulations is a better control measure since it allows controlling for short-term and long-term individual differences.

Nevertheless, whether it is homeostatic plasticity or the perception of pain and/or stress that causes the functional connectivity pattern should be addressed in further research by systematically recording subjective pain and stress levels during stimulation as well as pre-existing temporary brain states. Furthermore, since rMT is vastly variable inter- and intraindividually, and even a highly standardized procedure does not guarantee a reliable assessment, it is a problematic way of determining this stimulation parameter. Moreover, as we only used an intermittent theta burst protocol, it is unknown how stimulation protocol (e.g., low vs. high frequency, continuous vs. intermittent, etc.) and the current state of the network interact. Additionally, the number of subjects is rather low, which might weaken the conclusiveness of this pilot study.

Further research, incorporating a bigger sample size, different combinations of stimulation protocols (e.g., continues vs. intermittent theta burst) and intensities as well as sham stimulation, should be carried out to answer this question. Furthermore, studies comparing healthy participants and patients could further our understanding of the frontal-striatal network, its dysfunction and TMS as a possible treatment tool.

\section{Conclusions}

Even considering its relatively small number of subjects, this pilot study demonstrates that small differences in iTBS intensity applied to the cortex can lead to vastly different effects in brain activity of a connected functional network. The fact that fronto-caudal functional connectivity was significantly more synchronized after sub-threshold stimulation of the PFC, when compared to after supra-threshold stimulation, reveals the existence of highly complex communication mechanisms based on activation synchronization of connected areas.

\section{ETHICS STATEMENT}

This study was approved by and carried out in accordance with the recommendations of the ethical committee of the faculty of medicine of the RWTH Aachen University, Aachen, Germany (protocol number: 003/15). All subjects gave written informed consent in accordance with the Declaration of Helsinki.

\section{AUTHOR CONTRIBUTIONS}

Theoretical considerations, experimental design and experimental plan were prepared by FB, FM, and KS. The experimental setup and data collection was conducted by KS and IA. Data management and processing, statistical analysis and interpretation, as well as the manuscript preparation was carried out by IA. 


\section{FUNDING}

This work was supported by the Interdisciplinary Center for Clinical Research (IZKF) Aachen within the Faculty of Medicine at RWTH Aachen University.

\section{REFERENCES}

Angelucci, A., Bijanzadeh, M., Nurminen, L., Federer, F., Merlin, S., and Bressloff, P. C. (2017). Circuits and mechanisms for surround modulation in visual cortex. Annu. Rev. Neurosci. 40, 425-451. doi: 10.1146/annurev-neuro072116-031418

Arnsten, A. F. (2009). Stress signalling pathways that impair prefrontal cortex structure and function. Nat. Rev. Neurosci. 10, 410-422. doi: 10.1038/ nrn 2648

Aungst, J. L., Heyward, P. M., Puche, A. C., Karnup, S. V., Hayar, A., Szabo, G., et al. (2003). Centre-surround inhibition among olfactory bulb glomeruli. Nature 426, 623-629. doi: 10.1038/nature02185

Baggio, H. C., Segura, B., Garrido-Millan, J. L., Marti, M. J., Compta, Y., Valldeoriola, F., et al. (2015). Resting-state frontostriatal functional connectivity in Parkinson's disease-related apathy. Mov. Disord. 30, 671-679. doi: $10.1002 / \mathrm{mds} .26137$

Bakker, N., Shahab, S., Giacobbe, P., Blumberger, D. M., Daskalakis, Z. J., Kennedy, S. H., et al. (2015). rTMS of the dorsomedial prefrontal cortex for major depression: safety, tolerability, effectiveness, and outcome predictors for $10 \mathrm{~Hz}$ versus intermittent theta-burst stimulation. Brain Stimul. 8, 208-215. doi: 10.1016/j.brs.2014.11.002

Banks, S. J., Eddy, K. T., Angstadt, M., Nathan, P. J., and Phan, K. L. (2007). Amygdala-frontal connectivity during emotion regulation. Soc. Cogn. Affect. Neurosci. 2, 303-312. doi: 10.1093/scan/nsm029

Benjamini, Y., and Hochberg, Y. (1995). Controlling the false discovery rate: a practical and powerful approach to multiple testing. J. R. Stat. Soc. B 57, 289-300. doi: 10.1111/j.2517-6161.1995.tb02031.x

Benninger, D. H., Berman, B., Houdayer, E., Pal, N., Luckenbaugh, D., Schneider, L., et al. (2011). Intermittent theta-burst transcranial magnetic stimulation for treatment of Parkinson disease. Neurology 76, 601-609. doi: 10.1212/WNL.0b013e31820ce6bb

Beste, C., Ness, V., Lukas, C., Hoffmann, R., Stüwe, S., Falkenstein, M., et al. (2012). Mechanisms mediating parallel action monitoring in fronto-striatal circuits. Neuroimage 62, 137-146. doi: 10.1016/j.neuroimage.2012.05.019

Bestmann, S., Baudewig, J., Siebner, H. R., Rothwell, J. C., and Frahm, J. (2005). BOLD MRI responses to repetitive TMS over human dorsal premotor cortex. Neuroimage 28, 22-29. doi: 10.1016/j.neuroimage.2005.05.027

Bohning, D. E., Shastri, A., Wassermann, E. M., Ziemann, U., Lorberbaum, J. P., Nahas, Z., et al. (2000). BOLD-f MRI response to single-pulse transcranial magnetic stimulation (TMS). J. Magn. Reson. Imaging 11, 569-574. doi: 10.1002/1522-2586(200006)11:6<569::aid-jmri1>3.0.co;2-3

Bouyer, J., Park, D., Joh, T., and Pickel, V. (1984). Chemical and structural analysis of the relation between cortical inputs and tyrosine hydroxylase-containing terminals in rat neostriatum. Brain Res. 302, 267-275. doi: 10.1016/00068993(84)90239-7

Breitenstein, C., Daum, I., and Ackermann, H. (1998). Emotional processing following cortical and subcortical brain damage: contribution of the frontostriatal circuitry. Behav. Neurol. 11, 29-42. doi: 10.1155/1998/579029

Brunelin, J., Szekely, D., Costes, N., Mondino, M., Bougerol, T., Saoud, M., et al. (2011). Theta burst stimulation in the negative symptoms of schizophrenia and striatal dopamine release: an iTBS- $\left[{ }^{11} \mathrm{C}\right]$ raclopride PET case study. Schizophr. Res. 131, 264-265. doi: 10.1016/j.schres.2011.05.019

Choi, E. Y., Yeo, B. T., and Buckner, R. L. (2012). The organization of the human striatum estimated by intrinsic functional connectivity. J. Neurophysiol. 108, 2242-2263. doi: 10.1152/jn.00270.2012

Cohen, J. (2013). Statistical Power Analysis for the Behavioral Sciences. Abingdonon-Thames: Routledge.

Courtney, K. E., Ghahremani, D. G., and Ray, L. A. (2013). Fronto-striatal functional connectivity during response inhibition in alcohol dependence. Addict. Biol. 18, 593-604. doi: 10.1111/adb.12013

\section{ACKNOWLEDGMENTS}

We would like to thank Harshal Patel for his support with the fMRI protocols and measurements, as well as Antonio Pellicano for useful discussions about the statistical analyses.

Cubillo, A., Halari, R., Ecker, C., Giampietro, V., Taylor, E., and Rubia, K. (2010) Reduced activation and inter-regional functional connectivity of fronto-striatal networks in adults with childhood Attention-Deficit Hyperactivity Disorder (ADHD) and persisting symptoms during tasks of motor inhibition and cognitive switching. J. Psychiatr. Res. 44, 629-639. doi: 10.1016/j.jpsychires. 2009.11.016

Curtis, C. E., and D'Esposito, M. (2003). Persistent activity in the prefrontal cortex during working memory. Trends Cogn. Sci. 7, 415-423. doi: 10.1016/s13646613(03)00197-9

Di Martino, A., Scheres, A., Margulies, D. S., Kelly, A., Uddin, L. Q., Shehzad, Z., et al. (2008). Functional connectivity of human striatum: a resting state FMRI study. Cereb. Cortex 18, 2735-2747. doi: 10.1093/cercor/bhn041

Duprat, R., Desmyter, S., van Heeringen, K., Van den Abbeele, D., Tandt, H., Bakic, J., et al. (2016). Accelerated intermittent theta burst stimulation treatment in medication-resistant major depression: a fast road to remission? J. Affect. Disord. 200, 6-14. doi: 10.1016/j.jad.2016.04.015

Effnert, I., Sakreida, K., Binkofski, F., and Mottaghy, F. (2016a). "EP 71. The effect of intermittent theta burst stimulation over the dorsolateral prefrontal cortex on cortico-striatal connectivity," in 60th Annual Meeting of the German Society for Clinical Neurophysiology and Functional Imaging (DGKN) (Düsseldorf: Elsevier).

Effnert, I., Sakreida, K., Mottaghy, F., and Binkofski, F. (2016b). "P236 The effect of intermittent theta burst stimulation over the prefrontal cortex on resting-state fronto-striatal connectivity," in 6th International Conference on Transcranial Brain Stimulation (TBS) (Göttingen: Elsevier).

Fitzgerald, P. B., Maller, J. J., Hoy, K. E., Thomson, R., and Daskalakis, Z. J. (2009). Exploring the optimal site for the localization of dorsolateral prefrontal cortex in brain stimulation experiments. Brain Stimul. 2, 234-237. doi: 10.1016/j.brs. 2009.03.002

Fox, M. D., Halko, M. A., Eldaief, M. C., and Pascual-Leone, A. (2012). Measuring and manipulating brain connectivity with resting state functional connectivity magnetic resonance imaging (fcMRI) and transcranial magnetic stimulation (TMS). Neuroimage 62, 2232-2243. doi: 10.1016/j.neuroimage.2012. 03.035

Glenn, A. L., Raine, A., Schug, R. A., Young, L., and Hauser, M. (2009). Increased DLPFC activity during moral decision-making in psychopathy. Mol. Psychiatry 14, 909-911. doi: 10.1038/mp.2009.76

Heller, A. S., Johnstone, T., Shackman, A. J., Light, S. N., Peterson, M. J., Kolden, G. G., et al. (2009). Reduced capacity to sustain positive emotion in major depression reflects diminished maintenance of fronto-striatal brain activation. Proc. Natl. Acad. Sci. U S A 106, 22445-22450. doi: 10.1073/pnas. 0910651106

Huang, Y.-Z., Edwards, M. J., Rounis, E., Bhatia, K. P., and Rothwell, J. C. (2005). Theta burst stimulation of the human motor cortex. Neuron 45, 201-206. doi: 10.1016/j.neuron.2004.12.033

Janicak, P. G., and Dokucu, M. E. (2015). Transcranial magnetic stimulation for the treatment of major depression. Neuropsychiatr. Dis. Treat. 11, 1549-1560. doi: 10.2147/NDT.S67477

Johnson, K. A., Baig, M., Ramsey, D., Lisanby, S. H., Avery, D., McDonald, W. M., et al. (2013). Prefrontal rTMS for treating depression: location and intensity results from the OPT-TMS multi-site clinical trial. Brain Stimul. 6, 108-117. doi: 10.1016/j.brs.2012.02.003

Kanno, M., Matsumoto, M., Togashi, H., Yoshioka, M., and Mano, Y. (2003). Effects of acute repetitive transcranial magnetic stimulation on extracellular serotonin concentration in the rat prefrontal cortex. J. Pharmacol. Sci. 93, 451-457. doi: 10.1254/jphs.93.451

Kanno, M., Matsumoto, M., Togashi, H., Yoshioka, M., and Mano, Y. (2004). Effects of acute repetitive transcranial magnetic stimulation on dopamine release in the rat dorsolateral striatum. J. Neurol. Sci. 217, 73-81. doi: 10.1016/j. jns.2003.08.013 
Karreman, M., and Moghaddam, B. (1996). The prefrontal cortex regulates the basal release of dopamine in the limbic striatum: an effect mediated by ventral tegmental area. J. Neurochem. 66, 589-598. doi: 10.1046/j.1471-4159.1996. 66020589.x

Keck, M., Welt, T., Müller, M., Erhardt, A., Ohl, F., Toschi, N., et al. (2002). Repetitive transcranial magnetic stimulation increases the release of dopamine in the mesolimbic and mesostriatal system. Neuropharmacology 43, 101-109. doi: 10.1016/s0028-3908(02)00069-2

Lang, N., Siebner, H. R., Ernst, D., Nitsche, M. A., Paulus, W., Lemon, R. N., et al. (2004). Preconditioning with transcranial direct current stimulation sensitizes the motor cortex to rapid-rate transcranial magnetic stimulation and controls the direction of after-effects. Biol. Psychiatry 56, 634-639. doi: 10.1016/j. biopsych.2004.07.017

Leh, S. E., Ptito, A., Chakravarty, M. M., and Strafella, A. P. (2007). Fronto-striatal connections in the human brain: a probabilistic diffusion tractography study. Neurosci. Lett. 419, 113-118. doi: 10.1016/j.neulet.2007.04.049

Levy, B. J., and Wagner, A. D. (2011). Cognitive control and right ventrolateral prefrontal cortex: reflexive reorienting, motor inhibition and action updating. Ann. N Y Acad. Sci. 1224, 40-62. doi: 10.1111/j.1749-6632.2011. 05958.x

Li, C.-T., Chen, M.-H., Juan, C.-H., Huang, H.-H., Chen, L.-F., Hsieh, J.-C., et al. (2014). Efficacy of prefrontal theta-burst stimulation in refractory depression: a randomized sham-controlled study. Brain 137, 2088-2098. doi: 10.1093/brain/awu109

Li, C.-S. R., Ide, J. S., Zhang, S., Hu, S., Chao, H. H., and Zaborszky, L. (2014). Resting state functional connectivity of the basal nucleus of Meynert in humans: in comparison to the ventral striatum and the effects of age. Neuroimage 97, 321-332. doi: 10.1016/j.neuroimage.2014.04.019

Menzies, L., Chamberlain, S. R., Laird, A. R., Thelen, S. M., Sahakian, B. J., and Bullmore, E. T. (2008). Integrating evidence from neuroimaging and neuropsychological studies of obsessive-compulsive disorder: the orbitofrontostriatal model revisited. Neurosci. Biobehav. Rev. 32, 525-549. doi: 10.1016/j. neubiorev.2007.09.005

Murase, S., Grenhoff, J., Chouvet, G., Gonon, F. G., and Svensson, T. H. (1993). Prefrontal cortex regulates burst firing and transmitter release in rat mesolimbic dopamine neurons studied in vivo. Neurosci. Lett. 157, 53-56. doi: 10.1016/0304-3940(93)90641-w

Nahas, Z., Lomarev, M., Roberts, D. R., Shastri, A., Lorberbaum, J. P., Teneback, C., et al. (2001). Unilateral left prefrontal transcranial magnetic stimulation (TMS) produces intensity-dependent bilateral effects as measured by interleaved BOLD fMRI. Biol. Psychiatry 50, 712-720. doi: 10.1016/s00063223(01)01199-4

Oldfield, R. C. (1971). The assessment and analysis of handedness: the Edinburgh inventory. Neuropsychologia 9, 97-113. doi: 10.1016/0028-3932(71) 90067-4

Phelps, E. A., and LeDoux, J. E. (2005). Contributions of the amygdala to emotion processing: from animal models to human behavior. Neuron 48, 175-187. doi: 10.1016/j.neuron.2005.09.025

Robbins, T. W., and Everitt, B. J. (1992). Functions of dopamine in the dorsal and ventral striatum. Semin. Neurosci. 4, 119-127. doi: 10.1016/10445765(92)90010-y

Robinson, J. L., Laird, A. R., Glahn, D. C., Blangero, J., Sanghera, M. K., Pessoa, L., et al. (2012). The functional connectivity of the human caudate: an application of meta-analytic connectivity modeling with behavioral filtering. Neuroimage 60, 117-129. doi: 10.1016/j.neuroimage.2011.12.010

Rossi, S., Hallett, M., Rossini, P. M., Pascual-Leone, A., and Safety of TMS Consensus Group. (2009). Safety, ethical considerations, and application guidelines for the use of transcranial magnetic stimulation in clinical practice and research. Clin. Neurophysiol. 120, 2008-2039. doi: 10.1016/j.clinph.2009. 08.016

Rossini, P. M., Burke, D., Chen, R., Cohen, L., Daskalakis, Z., Di Iorio, R., et al. (2015). Non-invasive electrical and magnetic stimulation of the brain, spinal cord, roots and peripheral nerves: basic principles and procedures for routine clinical and research application. An updated report from an IFCN
Committee. Clin. Neurophysiol. 126, 1071-1107. doi: 10.1016/j.clinph.2015. 02.001

Salomons, T. V., Dunlop, K., Kennedy, S. H., Flint, A., Geraci, J., Giacobbe, P., et al. (2014). Resting-state cortico-thalamic-striatal connectivity predicts response to dorsomedial prefrontal rTMS in major depressive disorder. Neuropsychopharmacology 39, 488-498. doi: 10.1038/npp.2013.222

Sesack, S. R., and Pickel, V. M. (1992). Prefrontal cortical efferents in the rat synapse on unlabeled neuronal targets of catecholamine terminals in the nucleus accumbens septi and on dopamine neurons in the ventral tegmental area. J. Comp. Neurol. 320, 145-160. doi: 10.1002/cne.903200202

Siebner, H. R., Lang, N., Rizzo, V., Nitsche, M. A., Paulus, W., Lemon, R. N., et al. (2004). Preconditioning of low-frequency repetitive transcranial magnetic stimulation with transcranial direct current stimulation: evidence for homeostatic plasticity in the human motor cortex. J. Neurosci. 24, 3379-3385. doi: 10.1523/JNEUROSCI.5316-03.2004

Sohn, Y. H., and Hallett, M. (2004). Surround inhibition in human motor system. Exp. Brain Res. 158, 397-404. doi: 10.1007/s00221-004-1909-y

Strafella, A. P., Paus, T., Barrett, J., and Dagher, A. (2001). Repetitive transcranial magnetic stimulation of the human prefrontal cortex induces dopamine release in the caudate nucleus. J. Neurosci. 21, RC157-RC157. doi: 10.1523/JNEUROSCI.21-15-j0003.2001

Strafella, A. P., Paus, T., Fraraccio, M., and Dagher, A. (2003). Striatal dopamine release induced by repetitive transcranial magnetic stimulation of the human motor cortex. Brain 126, 2609-2615. doi: 10.1093/brain/awg268

Wang, Y., Zhu, J., Li, Q., Li, W., Wu, N., Zheng, Y., et al. (2013). Altered fronto-striatal and fronto-cerebellar circuits in heroin-dependent individuals: a resting-state fMRI study. PLoS One 8:e58098. doi: 10.1371/journal.pone. 0058098

Wessa, M., Houenou, J., Paillère-Martinot, M.-L., Berthoz, S., Artiges, E., Leboyer, M., et al. (2007). Fronto-striatal overactivation in euthymic bipolar patients during an emotional go/nogo task. Am. J. Psychiatry 164, 638-646. doi: 10.1176/appi.ajp.164.4.638

Yan, C.-G., Wang, X.-D., Zuo, X.-N., and Zang, Y.-F. (2016). DPABI: data processing and analysis for (resting-state) brain imaging. Neuroinformatics 14 339-351. doi: 10.1007/s12021-016-9299-4

Zafar, N., Paulus, W., and Sommer, M. (2008). Comparative assessment of best conventional with best theta burst repetitive transcranial magnetic stimulation protocols on human motor cortex excitability. Clin. Neurophysiol. 119, 1393-1399. doi: 10.1016/j.clinph.2008.02.006

Zhang, S., Hu, S., Chao, H. H., and Li, C. S. R. (2016). Resting-state functional connectivity of the locus coeruleus in humans: in comparison with the ventral tegmental area/substantia nigra pars compacta and the effects of age. Cereb. Cortex 26, 3413-3427. doi: 10.1093/cercor/bhv172

Zhang, S., Ide, J. S., and Li, C. S. R. (2011). Resting-state functional connectivity of the medial superior frontal cortex. Cereb. Cortex 22, 99-111. doi: 10.1093/cercor/bhr088

Ziemann, U., Paulus, W., Nitsche, M. A., Pascual-Leone, A., Byblow, W. D., Berardelli, A., et al. (2008). Consensus: motor cortex plasticity protocols. Brain Stimul. 1, 164-182. doi: 10.1016/j.brs.2008.06.006

Ziemann, U., and Siebner, H. R. (2015). Inter-subject and inter-session variability of plasticity induction by non-invasive brain stimulation: boon or bane? Brain Stimul. 8, 662-663. doi: 10.1016/j.brs.2015.01.409

Conflict of Interest Statement: The authors declare that the research was conducted in the absence of any commercial or financial relationships that could be construed as a potential conflict of interest.

Copyright (C) 2019 Alkhasli, Sakreida, Mottaghy and Binkofski. This is an open-access article distributed under the terms of the Creative Commons Attribution License (CC BY). The use, distribution or reproduction in other forums is permitted, provided the original author(s) and the copyright owner(s) are credited and that the original publication in this journal is cited, in accordance with accepted academic practice. No use, distribution or reproduction is permitted which does not comply with these terms. 